Volume 9, No.4, July - August 2020

International Journal of Advanced Trends in Computer Science and Engineering

Available Online at http://www.warse.org/IJATCSE/static/pdf/file/ijatcse134942020.pdf

https://doi.org/10.30534/ijatcse/2020/134942020

\title{
An Effective LSTM Network Model For Accurate Prediction of Delays in Indian Railway Networks
}

\author{
Lokaiah Pullagura $^{1}$, Dr. Anil Kumar ${ }^{2}$ \\ ${ }^{1}$ Research Scholar, Dept. of Computer Science \& Engineering, Sri Satya Sai University of Technology \& \\ Medical Sciences, Sehore, Bhopal-Indore Road, Madhya Pradesh, India \\ ${ }^{2}$ Research Guide, Dept. of Computer Science \& Engineering, Sri Satya Sai University of Technology \& \\ Medical Sciences, Sehore, Bhopal Indore Road, Madhya Pradesh, India
}

\begin{abstract}
Train delays can securely be viewed as a vital part of train travel in India giving the present delay patterns, yet what bothers the travellers most is the vulnerability around their train travel. Here we propose LSTM with delay as delayed connection memory, an anomaly detection strategy for time arrangement data. We first form a predictive model from typical training data, at that point perform anomaly detection depends on the prediction error for watched data. Be that as it may, there are multiple states in the waveforms of ordinary data, which may bring down prediction accuracy. To manage this issue, we use multiple prediction models dependent on delay connection memory for anomaly detection. In this plan, the prediction accuracy emphatically relies upon the strategy for choosing an appropriate predictive model from multiple potential models. We propose a novel strategy to determine the best possible predictive model for anomaly detection. Our methodology gives multiple anticipated worth competitors ahead of time and chooses the one that is nearest to the deliberate worth. We delay the model determination until the comparing estimated values are procured. Utilizing this idea for anomaly detection, LSTM chooses the best possible predictive model to improve prediction accuracy. In our exploratory assessment utilizing genuine and artificial data, delay connection distinguishes inconsistencies more precisely than strategies in correlation.
\end{abstract}

Key words : Anomaly detection, Deep learning, LSTM, Time-series data

\section{INTRODUCTION}

Multiple factors related with the run of a train can influence the appearance time of a train at the station and travellers are regularly left hanging tight for a considerable length of time before their train at long last shows up. The outcome; ceaseless tension of travellers, many worker hours squandered, and pointless blockage at all the stations. RailYatri (figure 1), a travel fire up, has advanced an exceptional Estimated Arrival Time (ETA) prediction algorithm utilizing Machine Learning and Statistical Modelling techniques to foresee the appearance time of running trains at their forthcoming stoppage with much better exactness. The algorithm has been trained to break down authentic data of train runs spread over numerous years and anticipate future results.

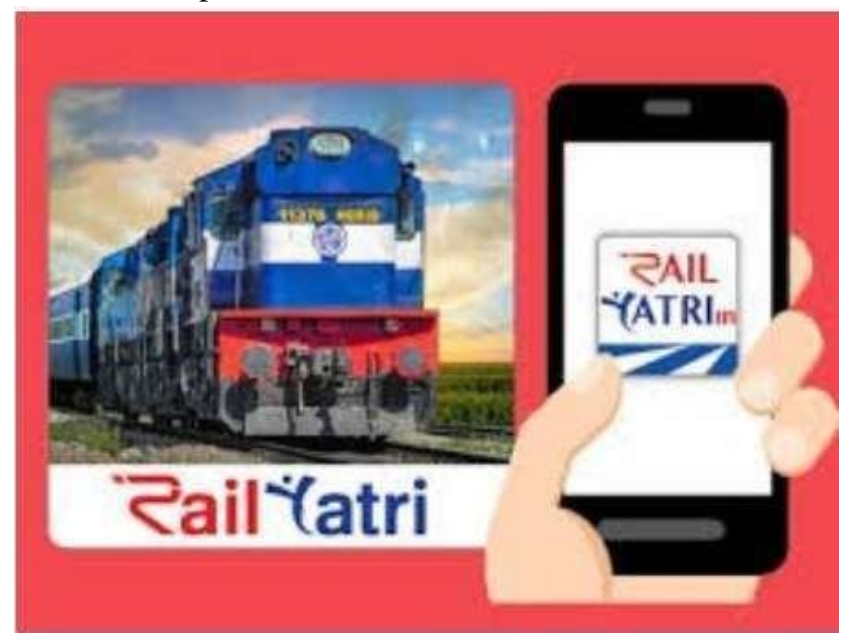

Figure 1: Rail Yatri Machine learning to sharpen train delays

Train delays can securely be viewed as a vital part of train travel in India giving the present delay patterns, yet what troubles the travellers most is the vulnerability around their train travel. Studies show that while train travellers have submitted to delays being a piece of their travel, their disappointment emerges from the powerlessness of the current systems to effectively direct them on the evaluated time of appearance (ETA) of their trains. This leaves them standing by interminably at stages with no thought of the specific time of appearance of their trains.

As of late, accomplished neural network has plenty of achievements and have been at the centre of attention. They are broadly utilized for design acknowledgment, arrangement, and relapse in different fields, for example, PC vision, regular language preparation, and large data examination. Connections among neurons assume a vital job 
in Neural Networks on the grounds that they regulate the data stream in the Neural Networks. Based on connection By and large, Neural Networks are separated into two classes as indicated via their usage.
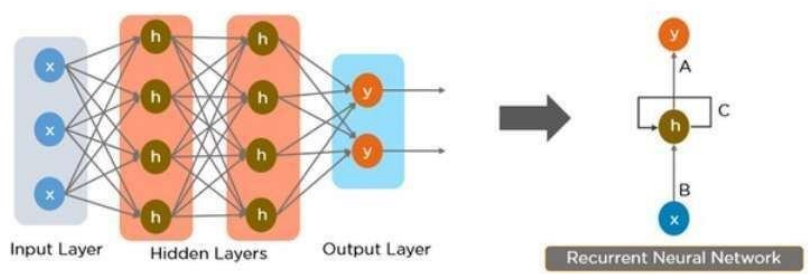

Figure 2: General Recurrent neural network

This unit of LSTM proposes an exceptional connection associated with exponential activity. They can light up both data battle and yield conflict gives well. Such multiplicative associations furthermore have been used in other top tier intermittent models; Long Short-Term Memory model is loosened up with opening associations with gate units associated in memory neurons. Gotten together with supplementary gate units, as such multiplicative associations progress a setting complex instrument to control the "read" else "state" strategy in respective memory neurons.

\section{LITERATURE REVIEW}

Martin Längkvist Proposed [1] in Feb 2015 There is an extending eagerness for the machine learning network to therefore pick up incorporate depictions honestly from the (unlabelled) data instead of using hand-organized features. The auto encoder is one procedure that can be used consequently. Regardless, for data sets with an elevated level of disturbance, a great deal of as far as possible in the auto encoder is used to constrain the changing blunder for these boisterous wellsprings of information. This paper proposes a procedure that improves the segment learning process by focusing on the task appropriate data in the data. This specific thought is practiced by weighting the amusement mistake and diminishing the effect of boisterous commitments during the learning methodology. The proposed model is prepared on different transparently available picture data sets and the test blunder rate is appeared differently in relation to a standard small auto encoder and various methodologies, for instance, the denoising auto encoder and contractive auto encoder.

Tianyu Song [2] in March 2020 LSTM shows its commonness in discharge re-authorizations, and the ML building is as huge as the ML approach. Moreover, a subfield of ML, Deep Learning (DL) is logically stressed over datasets, calculations and layered structures. In this examination, a framework is proposed to assess defencelessness duties of the model set, ML approach, ML building and their relationship to multi-step time-game plan envisioning subject to the assessment of distinction (ANOVA) speculation. The results exhibit that none of the researched powerlessness sources are immaterial and the effect of defencelessness sources vacillates with lead times and discharges. By then a discharge re-sanctioning, using Recurrent Neural Networks (RNNs), is taken for example. Long Short-Term Memory (LSTM) organize, a top tier DL approach, was picked as a result of its unprecedented introduction in time-course of action checking, and differentiated and direct RNN. As a dynamic device provoking liberal changes over various regions, Machine Learning (ML) methods have gotten creating thought in the field of hydrology on account of their conceivable outcomes to appraise time course of action. The proposed structure can both reveal defencelessness estimation in ML/DL showing and offer references to ML approach evaluation and designing arrangement in discharge proliferations. Regardless of different employments of novel ML/DL methods in discharge propagation, the powerlessness related with ML/DL exhibiting has not drawn a great deal of thought, in spite of the way that it is a critical issue. Furthermore, novel discharge checking building is arranged by combining the inclination of hydrology and stacked DL structure, and differentiated and standard structure.

Yong Yu [3] expressed on May 2019, At long last, future research headings are introduced for LSTM networks. We survey the LSTM cell and its variations to investigate the learning limit of the LSTM cell. The LSTM has become the focal point of profound learning. Besides, the LSTM networks are partitioned into two general classes: LSTMoverwhelmed networks and incorporated LSTM networks. Since its presentation, practically all the energizing outcomes dependent on RNNs have been accomplished by the LSTM. Be that as it may, RNNs comprising of sigma cells or tan cells can't get familiar with the significant data of info data when the information hole is huge. What's more, their different applications are talked about. By bringing gate capacities into the phone structure, the long short-term memory (LSTM) could deal with the issue of long-term conditions well. Recurrent neural networks (RNNs) have been generally received in examine zones worried about successive data, for example, content, sound, and video.

\section{PROPOSED METHODOLOGY}

The Recurrent Neural Network is appropriate for consecutive data and has been broadly applied in the field of NLP. In particular, LSTM network is very impressive Recurrent NN with an increasingly unpredictable cell structure and defeats the angle evaporating issue in Recurrent Neural Networks. 
We consider an info arrangement $\mathrm{x}=[\mathrm{x} 1, \mathrm{x} 2, \ldots, \mathrm{xT}]$, and the Long Short Term Memory model processes the shrouded state $\mathrm{h}=[\mathrm{h} 1, \mathrm{~h} 2, \ldots, \mathrm{hT}]$ for each time step and gets yield grouping $\mathrm{y}=[\mathrm{y} 1, \mathrm{y} 2, \ldots, \mathrm{yT}]$.

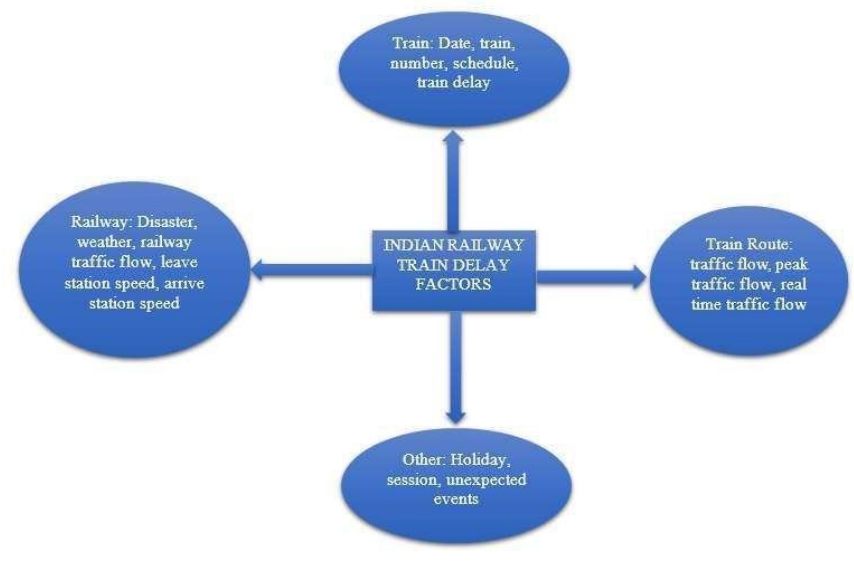

Figure 3: Train delay factors

Figure. 4 shows that the single Long Short Term Memory cell contains four gate structures, to be specific overlook gate, competitor gate, input gate, and yield gate. The shrouded layer work $\mathrm{H}$ is executed by the accompanying capacities spoke to by the gates:

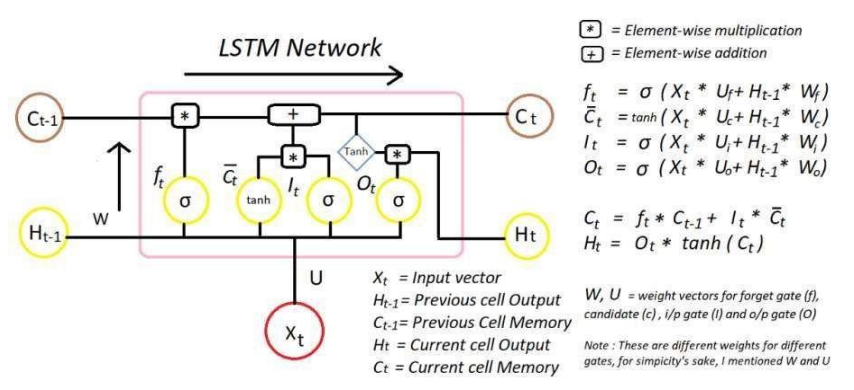

Figure 4: Gates structured with LSTM cell

where $\sigma$ is the sigmoid of the activation work and $\mathrm{ft}$ is the overlook gate that selects the data to be deleted from the last cell state. It and Ct separate the information gateway and the requesting gateway and together select the data to be included in the new status of the cell from the current status of the cell. Ot is the yield gate that selects the degree of discovery of the status of the phone. The structure stacked by the Long Short Term Memory cells simply appears as a slope path that allows the angle to flow from upstream to downstream without interruption during the return. The first is a standard design based on LSTM, the second is connected to a fully assigned layer, and the third is connected to an additional layer. The data was pre-processed to create a data set from messages from the training and testing process.
The defer factors look into LSTM model Figure 5 calculation and produce the memory square yield ht, while the no-postpone factors meet with ht to outline new data. By then, a feed-forward neural system is developed for the new information. Figure 5 shows the improved LSTM model computing strategy. One continuously concealed layer 2 along with overhaul the non-linear verbalization limit of a system. The data factors are divided into the defer factors and no-postpone factors. Henceforth, the neurons number and the trade limit of shrouded layer 2 should be suitably has to set the time to control the usage.

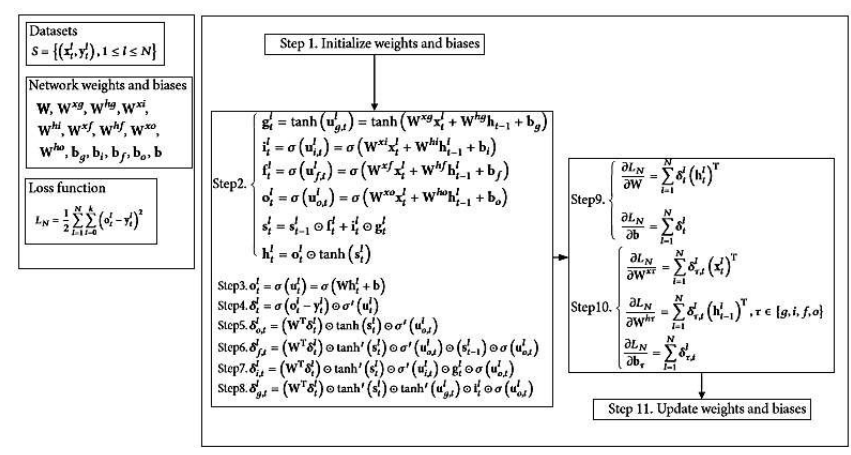

Figure 5: LSTM basic Back propagation flow inside the memory block

The defer factors look into LSTM model Figure 5 calculation and produce the memory square yield ht, while the no-postpone factors meet with ht to outline new data. By then, a feed-forward neural system is developed for the new information. Figure 5 shows the improved LSTM model computing strategy. One continuously concealed layer 2 along with overhaul the non-linear verbalization limit of a system. The data factors are divided into the defer factors and no-postpone factors. Henceforth, the neurons number and the trade limit of shrouded layer 2 should be suitably has to set the time to control the usage. So, the no-postpone factors can direct use developing variable time $\square$ finally without complex change and the kind of delayed consequence. The LSTM which is an improved model also can save getting ready time considering the way that, for nopostpone factors, there are absence of prerequisite for discontinuous inside estimation of the memory square. Regardless, it extends the time usage with extra shrouded second layer.

Can be expressed as:

$$
\begin{aligned}
& L \mathrm{~N}=\frac{1}{2} \sum_{l=1}^{N}\left(o_{k}^{l}-\mathrm{y}_{l}\right)_{2} \\
& \sigma(\mathrm{u} l k, 1)=\sigma([\mathrm{Wh} l k \mathrm{~W} n \mathrm{r} l]+[\mathrm{bb} n])
\end{aligned}
$$


Where 1 represents no-delay variable matrices; Wn and bn speak to the no-delay variables weight and bias matrix individually; ulk, 1 indicates the non-initiated yield of hidden layer 1. Given the extra hidden second layer, following two stages are communicated as:

$\mathrm{o} l k=\sigma(\mathrm{u} l k, 2)=\sigma(\mathrm{Wout} \sigma(\mathrm{u} l k, 1)+\mathrm{bout})$

$\delta l k=\sigma(\mathrm{ol} k-\mathrm{yl} k) \odot \sigma(\mathrm{u} l k, 2)$

Where $\mathrm{W}_{\text {out }}$ represent the weight and $\mathrm{b}_{\text {out }}$ bias of the output layer matrix, respectively; ulk, 2 represents the response of hidden non-activated layer.

\section{EXPERIMENTAL RESULT}

Below Experiment is used to analyse the effects of the deep Recurrent Neural Network architectures. Using Recurrent Neural Network and the individual train delay neural network architecture, we will implement the daily model. First and Foremost we would be training daily status model via deep Recurrent Neural Network with different parameters followed by respective train delay model. Real data are collected from Indian Railways through cloud platform data available over is utilized. Daily status model and random particular train delay models are combined to get an effective LSTM network model for accurate prediction of delays in Indian railway networks.

\section{Daily status model and Random particular train delay} model:

Indian Railway data was used to Daily status model for analysis of departure delay. Two datasets were used with length parameter and delay threshold parameter value. One of the set used is sequence of 14 days with 30 minutes parameter threshold value and the next set is 18 days of sequence and 55 minutes of threshold value. For above two cases, varied architecture holding deep inputs hidden nodes along with Dataset of Recurrent Neural network and combined form is explained in next section. In the two cases, deep contribution to-shrouded design improved the exactness marginally. Highlights extricated from every datum test are taken care of into learning calculations. We later conclude to these calculations as "shallow model" calculations since they comprise of not many layers of creation. These incorporate Artificial Neural Network (ANN) with one shrouded layer also. Furthermore, by blending every single deep design, we could get 5-7\% improved correctness's contrasted with shallow ones. This implies we can get a genuinely precise defer status for a solitary day. Another perception was that the deep architectures were improving the exactness of the model. The consolidated engineering is utilizing deep contribution to-covered up, stacked RNN, and deeply covered up to yield architectures out and out. As a source of the perspective model, a shallow model was likewise tried. The shallow model incorporates a solitary LSTM layer and info and yield are straightforwardly connected to the LSTM layer. Table 1 shows the exactness's for various deep architectures of RNN.

By stacking numerous LSTM cells, the multifaceted nature of the model is expanding excessively and causing numerical challenges. In any event, for some other parameter settings, the stacked LSTM didn't meet numerically. One last perception from the trial is that the model is a superior Indicator for the bigger postpone edge esteem. This can be seen that the littler limit worth would be noisier than bigger limit esteem so it is more earnestly to anticipate littler edge esteem delays. At the end of the day, the bigger limit esteem is arranging defer days with a progressively positive standard. Anyhow, stacked RNN doesn't generally ensure an improvement. In the last trial case, it shows an improvement yet it doesn't in the primary case. It very well may be investigated that its intricacy makes the calculation of the model troublesome. It has been generally seen in RNN architectures with long successions.

Table 1: Daily status Model of Predictive delay analysis Using RNN

\begin{tabular}{|l|l|l|}
\hline \multicolumn{1}{|c|}{ Variables } & \multicolumn{1}{|c|}{$\begin{array}{c}\text { Number of } \\
\text { consecutive days: } \\
\text { 14 days } \\
\text { Threshold: } \mathbf{3 0} \\
\text { mints }\end{array}$} & $\begin{array}{c}\text { Number of } \\
\text { consecutive days: } \\
\text { 18 days } \\
\text { Threshold: } 55 \\
\text { mints }\end{array}$ \\
\hline RNN Data & 78.55 & 87.07 \\
\hline Input hidden nodes & 77.01 & 89.85 \\
\hline Shallow model & 79.65 & 90.12 \\
\hline $\begin{array}{l}\text { ELNM(combined } \\
\text { model) }\end{array}$ & $\mathbf{8 0 . 5 4}$ & $\mathbf{9 0 . 4 5}$ \\
\hline
\end{tabular}

Table 2: Accuracy of Single Train delay model

\begin{tabular}{|c|c|c|c|}
\hline Datasets & $\begin{array}{l}\text { No of nodes } \\
\text { hidden in } \\
\text { each Layers }\end{array}$ & Time-lapse & $\begin{array}{l}\text { Prediction } \\
\text { Accuracy }\end{array}$ \\
\hline 1 & 133 & 22 & 87.45 \\
\hline 2 & $133 \# 100$ & 22 & 87.65 \\
\hline 4 & $\begin{array}{l}133 \# 200 \text { \# } \\
15\end{array}$ & 22 & 87.89 \\
\hline 5 & $\begin{array}{l}133 \# 300 \# 200 \\
\# 100 \# 5\end{array}$ & 238 & 89.12 \\
\hline 5 (SMALL) & $\begin{array}{l}\text { 133\#300\#200 } \\
\# 100 \# 15\end{array}$ & 238 & 89.15 \\
\hline
\end{tabular}


Python pyplot.bar() bar graph is produced as shown in figure 6 for better data visualization using below python coding with matplotlib library Imported.

from matplotlib import pyplot as plot

import numpy as np

data $=[87.07,89.85,90.12,90.45]$,

$[78.55,77.01,79.65,80.54],] \mathrm{X}$-axis $=$ np.arange $(4)$

fig = plt.figure ()

axis=fig.add_axes $([0,0,1,1])$

axis.bar $(\mathrm{X}$-axis +0.00 , data[0], color $=$ 'g', width $=0.25)$

axis.bar(X-axis +0.25 , data[1], color $=$ 'b', width $=0.25)$ plot.show()
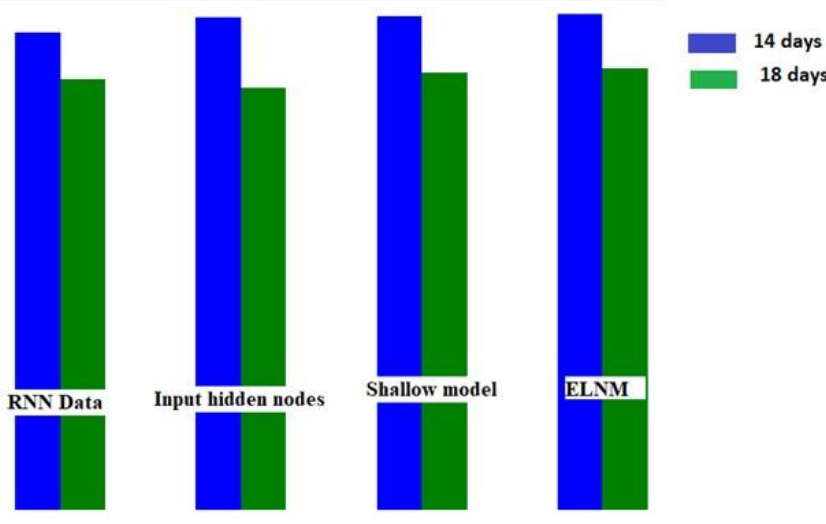

Figure 6: Bar graph data visualization of our Model using PyCharm

Effective LSTM Network Model [ELNM] for accurate prediction of delays in Indian railway networks by combining above two models:

We can make an effective LTSM network model for accurate prediction of delay using RNN in Indian Railway line by combination of above discussed models. Daily status model and random particular train delay models, where to be noted Daily status models uses the day to day status and Random particular delay model uses past analysed data structure. In the deep layered completely associated hubs, the quantity of layers, the quantity of shrouded hubs in each layer, duration, and the group size is fluctuated and the precision is tried. A duration implies one full go through the preparation set. At each emphasis, the quantity of tests utilized for the preparation is the clump size. Table 2 shows the correctness's obtained for various settings. From the outcomes, the deep model accomplished high exactness running from $86 \%$ to $87 \%$. This proposes deep learning approaches and models will perform better later on by gathering more information. Furthermore, the precision for the model tried in this part may be utilized for the immediate comparison with past investigations. In this way, $87.42 \%$ precision is the best exactness so far contrasting and the past best exactness $83.4 \%$. It likewise shows that the expanded number of layers is adding to improve precision. In this way, the deep learning way to deal with the Indian Railway train delay prediction is effective. Another perception is that more data with increasing ages of our combined model make the prediction progressively precise which is a typical perception in the vast majority of the past information examination errands.

\section{CONCLUSION}

In this paper, we looked at Neural Network's multiplicative as well as delayed connection, and here proposed another connection with delay for LSTM networks. RNN along with DCM units have outperformed the first four recurrent models in their class in several clusters with tasks also achieved consistent and efficient Machine learning, while this proposed offset relation in the DCM element has greatly enhanced the recurrent unit's ability to learn given the decrease in RNN learning inclination problems. In the event of such a complication allows to learn the dynamic transient statistics conditions in a system that illustrates the assignments. We 're creating a different recurring model called DCM. That, however, poses digital testing difficulties. The LSTM model and the DCM model are moreover unpredictable recurring models. In future research, we may want to reduce the complexity of repeated patterns while safeguarding their learning capacity.

\section{REFERENCES}

1. M. Langkvist and A. Loutfi. Learning feature representations with a cost-relevant sparse autoencoder, Int. J. Neural Syst. 25(1) (2015) 1450034.

2. Tianyu Song. Uncertainty Quantification in Machine Learning Modeling for MultiStepTime Series Forecasting: Example of Recurrent Neural Networks in Discharge Simulations, Water 2020, 12, 912. https://doi.org/10.3390/w12030912

3. Yong Yu. A Review of Recurrent Neural Networks: LSTM Cells and Network Architectures, May 2019 31(1):1-36.

4. Q. Guo, J. Jia, G. Shen, L. Zhang, L. Cai and Z. Yi. Learning robust uniform features for cross-media social data by using cross autoencoders, Knowl.Based Syst. 102 (2016) 64-75.

5. A. Panakkat and H. Adeli. Neural network models for earthquake magnitude prediction using multiple seismicity indicators, Int. J. Neural Syst. 17(1) (2007) 13-33.

6. H. Adeli and A. Karim. Fuzzy-wavelet RBFNN model for freeway incident detection, J. Transp. Eng. 126(6) (2000) 464-471.

7. A. Karim and H. Adeli. Comparison of fuzzy-wavelet radial basis function neural network freeway 
incident detection model with california algorithm, $\mathrm{J}$. Transp. Eng. 128(1) (2002) 21-30.

8. A. Karim and H. Adeli. Radial basis function neural network for work zone capacity and queue estimation, J. Transp. Eng. 129(5) (2003) 494-503.

9. S. Ghosh-Dastidar, H. Adeli and N. DaDCMehr. Principal component analysis-enhanced cosine radial basis function neural network for robust epilepsy and seizure detection, IEEE Trans. Biomed. Eng. 55(2) (2008) 512-518. https://doi.org/10.1109/TBME.2007.905490

10. Q. Fu, J. Wang, L. Zhang, Z. Yi and X. Fu. Computational models of implicit sequence learning: Distinguishing abstract processes from chunking processes, in Advances in Computational Psychophysiology (Science/AAAS, Washington, 2015), pp. 22-24.

11. A. Panakkat and H. Adeli. Recurrent neural network for approximate earthquake time and location prediction using multiple seismicity indicators, Comput.-Aided Civ. Infrastruct. Eng. 24(4) (2009) 280-292.

12. S. Ghosh-Dastidar and H. Adeli. Spiking neural networks, Int. J. Neural Syst. 19(4) (2009) 295-308.

13. Nawaf Alharbe, Fawaz Alharbi. Growing SelfOrganizing Map through a Hybrid Algorithm of Load Prediction, International Journal of Advanced Trends in Computer Science and Engineering,Volume 9, No.1, January - February 2020. https://doi.org/10.30534/ijatcse/2020/15912020

14. Dr.A.Nagarajan, J. Vasanth Wason. Machine Learning Approach to Predict Lung Cancer using CT scan Images, International Journal of Advanced Trends in Computer Science and Engineering,Volume 8, No.6, November - December 2019. https://doi.org/10.30534/ijatcse/2019/48862019

15. Vengatesan K, E Saravana Kumar, S. Yuvaraj, Punjabi Shivkumar Tanesh, Abhishek Kumar. An Approach for Remove Missing Values in Numerical and Categorical Values Using Two Way Table Marginal Joint Probability, International Journal of Advanced Science and Technology, Vol. 29, No. 5, (2020), pp. $2745-2756$.

16. Vengatesan K, S. Yuvaraj, Sayyad Samee, Punjabi Shivkumar Tanesh, Abhishek Kumar. Prediction of the Petrol Consumptions by using Data Mining Decision Classifier Classification Algorithm, TEST Engineering and Management, March - April 2020, ISSN: 0193-4120 Page No. 22206 - 22212. 